\title{
Do rats use shape to solve "shape discriminations"?
}

\author{
Loredana Minini ${ }^{1,2}$ and Kathryn J. Jeffery \\ ${ }^{1}$ Institute of Behavioural Neuroscience, Department of Psychology, University College London, \\ London WC1H OAP, United Kingdom
}

\begin{abstract}
Visual discrimination tasks are increasingly used to explore the neurobiology of vision in rodents, but it remains unclear how the animals solve these tasks: Do they process shapes holistically, or by using low-level features such as luminance and angle acuity? In the present study we found that when discriminating triangles from squares, rats did not use shape but instead relied on local luminance differences in the lower hemifield. A second experiment prevented this strategy by using stimuli-squares and rectangles-that varied in size and location, and for which the only constant predictor of reward was aspect ratio (ratio of height to width: a simple descriptor of "shape"). Rats eventually learned to use aspect ratio but only when no other discriminand was available, and performance remained very poor even at asymptote. These results suggest that although rats can process both dimensions simultaneously, they do not naturally solve shape discrimination tasks this way. This may reflect either a failure to visually process global shape information or a failure to discover shape as the discriminative stimulus in a simultaneous discrimination. Either way, our results suggest that simultaneous shape discrimination is not a good task for studies of visual perception in rodents.
\end{abstract}

Discrimination tasks have long been used to probe perceptual processes in animals, and visual discrimination tasks, particularly automated ones, are increasingly used in rodents because of the contribution that rodent neurobiological studies (such as newly emerging transgenic models) can make to understanding the brain mechanisms underlying visual perception (Bussey et al. 1994, 2001; Cook et al. 2004). Visual discrimination tasks often require the animals to choose between simple geometric shapes, and an implicit assumption in these studies is that rats and mice process shape in a holistic manner when making these discriminations. This assumption, however, has never been properly verified, and rests mainly on a relatively old literature (Fields 1932; Lashley 1938; Sutherland 1961a,b). Discovering the perceptual and cognitive processes underlying these kinds of behavioral tasks is critical if we are to make appropriate links with emerging neurobiological discoveries.

The present paper reports a series of investigations that led us to try and determine whether rats really use "shape" when making shape discriminations. Believing at the outset that this was a well-secured fact, we began with a more ambitious experiment to determine whether rats could respond to figures defined by illusory contours (the so-called "Kanizsa figures"), in which illusory form is created by means of "inducing stimuli" (Fig. 1) and is perceived in the absence of real (i.e., luminance-defined) contours. Several authors have proposed that the perception of illusory figures is largely the result of low-level mechanisms that have evolved to extract contours that are present in the environment but absent from the retinal representation: due, for instance, to occlusion or camouflage (Peterhans and von der Heydt 1991). Since this is a capability of great neurobiological importance, it is of interest to know if it is general across mammals and whether it is thus a fitting subject for, among other things, rodent transgenic studies. We thus set out to determine whether rats that had learned a discrimination based on luminancedefined shapes (squares and triangles) could transfer this responding to the same shapes made from illusory contours.

'Present address: Department of Physiology, Anatomy, and Genetics, University of Oxford, Oxford OX1 3PT, United Kindgom. ${ }^{2}$ Corresponding author.

E-mail lori.minini@physiol.ox.ac.uk; fax 44-1865-272543.

Article published online before print. Article and publication date are at http:// www.learnmem.org/cgi/doi/10.1101/lm.84406.
Our results showed that the animals could not in fact make this transfer, and in exploring the reason for this we were led to question whether they could transfer to shapes that were altered in other less complex ways, such as in luminance contrast and orientation. With a series of probe trials we finally determined that in making apparent shape discriminations, the rats in this first series of tests were really not processing shape at all, but using a set of low-level features that we had not anticipated: specifically, the luminance of the lower half of the figures.

This unexpected finding led us to set aside the question of illusory contour processing and to ask the much simpler question of whether rats use global shape at all when making so-called shape discriminations. In the case of visual forms, this turned out to be a difficult matter to address, because most shapes differ in local features as well as their global shape. For example, triangles differ from squares not only in their "triangularity" (Fields 1932), but also in the fact that their corner angles are more acute and there are fewer of them. However, reasoning that the core of the concept of "shape" must necessarily involve, at the very least, metric processing in two dimensions, we devised a set of stimuli in which the only relevant discriminand was information extracted from both dimensions simultaneously-that is, the aspect ratio (ratio of height to width). Rats were required to distinguish between squares (aspect ratio of 1) and rectangles (aspect ratio of 2 or 0.5 ), but the shapes were varied in absolute size, relative size, luminance, and allocentric position, to thwart any attempts to use local features.

As it happened, animals that were intended to use aspect ratio in fact began by trying to use a luminance-based strategy, based on an inadvertent confound we had introduced into the stimulus set; namely, because the squares were narrower than the rectangles on half the trials and wider on the remainder, and similarly with height, the task could be solved by choosing/ avoiding both very large and very small squares. When we foiled this with the addition of yet more stimuli, so that aspect ratio now became, as far as we could determine, the only remaining discriminative stimulus, these animals eventually recovered their discrimination to levels above chance, but this took thousands of trials, and performance remained poor even at asymptote. Control animals for which aspect ratio was always an optional discriminand (because the discrimination also had a unidimensional solution) did not use it at all. Taken together, the results 


\begin{tabular}{|c|c|c|c|}
\hline \multicolumn{4}{|c|}{ Experiment 1} \\
\hline Phase & Purpose & Stimuli & \\
\hline \begin{tabular}{|l|} 
Training \\
\end{tabular} & $\begin{array}{l}\text { To establish a basic shape } \\
\text { discrimination }\end{array}$ & $\begin{array}{l}\text { Square vs triangle } \\
\text { (with shapes on } \\
\text { black background) }\end{array}$ & 7 \\
\hline \begin{tabular}{|l|} 
Probe 1 \\
\end{tabular} & $\begin{array}{l}\text { To test transfer of responding } \\
\text { to the same shapes defined by } \\
\text { illusory contours }\end{array}$ & Kanizsa shapes & 4 \\
\hline Probe 2 & $\begin{array}{l}\text { To test transfer of responding } \\
\text { to altered but luminance-defined } \\
\text { (non-illusory) stimuli }\end{array}$ & $\begin{array}{l}\text { Outline shapes } \\
\text { Contrast shapes }\end{array}$ & $\begin{array}{ll}\nabla & \square \\
\nabla & \square\end{array}$ \\
\hline Probe 3 & $\begin{array}{l}\text { To test transfer of responding } \\
\text { to the training stimuli at altered } \\
\text { orientations }\end{array}$ & $\begin{array}{l}\text { Rotated shapes (subset). } \\
\text { Bottom ( } 30 \text { deg rotation) } \\
\text { but not top ( } 26.25 \mathrm{deg} \\
\text { rotation) shapes } \\
\text { elicited reversal of shape } \\
\text { preference in one subject }\end{array}$ & $30 \mathrm{deg}$ \\
\hline Probe 4 & $\begin{array}{l}\text { To test the possibility that rats } \\
\text { used lower hemifield features }\end{array}$ & $\begin{array}{l}\text { Reflected-diamond } \\
\text { Triangle-diamond } \\
\text { Reflected-square }\end{array}$ & $\overline{7}$ \\
\hline Probe 5 & $\begin{array}{l}\text { To test the possibility that rats } \\
\text { used lower hemifield luminance }\end{array}$ & $\begin{array}{l}\text { Displaced-square } \\
\text { Luminance } \\
\text { Displaced-triangle } \\
\text { Displaced-both }\end{array}$ & $\nabla$ \\
\hline
\end{tabular}

Figure 1. Summary of the training phases and stimuli used in Experiment 1. For Probe 3, the two stimulus sets are shown between which performance for one rat switched its preference from one shape to the other. The big change in behavior for such an apparently trivial stimulus change suggested that the animal might be using a local luminancebased strategy.

indicate that when presented with a discrimination between squares and rectangles, the animals did not use the "squareness" or "rectangleness" of the shapes, as a human would, but tried to solve the tasks using unidimensional size, or luminance (global or local). These results will be discussed in terms of the question of how rats process shapes, and of the utility of simultaneous discrimination tasks in probing the perceptual capabilities of rodents.

\section{Results}

\section{Experiment 1}

In the first experiment, rats were initially trained to discriminate between a square and a triangle (Fig. 1, Training), before proceeding to test trials in which the ability to transfer to transformed shapes was assessed (Fig. 1, Probes). Discrimination performance was measured as a mean percent correct performance, and was calculated as the percentage of correct responses over the total number of trials. Learning was considered complete if the animals reached a criterion of $85 \%$ over two consecutive sessions. Figure 2A shows the group learning curves during the training trials that continued into the test phase. It can be clearly seen that good discrimination performance was obtained with these stimuli and was maintained during the testing phase. The two rats trained with the square as the positive shape reached the criterion in a relatively small number of sessions (25 and 18 for rats 4 and 5, respectively). Subjects with the triangle as the posi- tive shape also learned the discrimination, although rats 1 and 3 reached asymptotic performance at $80 \%$ and rat 2 at $70 \%$. These animals also required a higher number of sessions $(52,61$, and 49 , respectively), indicating perhaps a greater difficulty in using this shape as the positive stimulus.

\section{Probe 1: A test for transfer to illusory figures}

Having learned the basic square-triangle discrimination, the rats were then given probe trials to see if they could transfer this discrimination to Kanizsa shapes formed from illusory contours. The rats were presented with Kanizsa shapes (Fig. 1, Probe 1) intermingled with the training stimuli. A total of 50 test trials were collected for each rat over the five test sessions for each of the two test stimuli. Figure $2 \mathrm{~B}$ shows individual mean test performance in the two test conditions. It can be seen that mean performance for all animals was close to chance level for the Kanizsa shapes, with the exception of rat 1 who seemed to have, if anything, a preference for the negative Kanizsa shape. A $t$-test confirmed that performance did not differ from chance $\left(t_{(4)}=-0.17, P=\mathrm{NS}\right)$. Taken together, these results indicate that these subjects did not transfer their discriminative performance to the Kanizsa stimuli.

Why did the animals not generalize to the Kanizsa stimuli? One possible explanation is that they did not perceive the Kanizsa square and triangle, perhaps because they lack the necessary visual processing capability to extract form from illusory contours. An alternative possibility, however, is that they simply had difficulty generalizing to stimuli that looked very different from the training set; for example, because of the change in the contrast polarity of the background that was introduced with these stimuli. In agreement with this account, both Fields (1932) and Lashley (1938) failed to find transfer from white shapes on a black background to stimuli with inverted contrast polarity. In a probe test, we explored generalization further by testing whether the animals would transfer performance on the learned shapes to altered but still luminance-defined (i.e., non-illusory) versions of the same shapes.

\section{Probe 2: Tests for transfer to altered stimuli}

The rats were tested with Outline stimuli, in which the shapes were created from white borders, and with Contrast stimuli, in which the contrast polarity was reversed so that the shapes became black on white. Figure $2 \mathrm{~B}$ shows individual performance with the Outline stimuli. It can be seen that all rats were able to solve the discrimination above chance level. A $t$-test confirmed that this performance was significant $\left(t_{(4)}=5.17, P<0.01\right)$. These results are in agreement with Lashley (1938), who also reported positive transfer to outline stimuli, and suggest that these rats could generalize to novel luminance-defined stimuli. They also rule out the possibility that these rats learned to respond to the specific luminance distribution (the retinal snapshot) of the training stimuli, as this was changed in the Outline stimuli without disruption of performance.

Performance on the Contrast stimuli, on the other hand, was greatly decreased (Fig. 2C). Repeated measures $t$-tests (twotailed) confirmed that this difference was highly significant $\left(t_{(11)}=15.38, P<0.001\right.$ and $t_{(10)}=16.98, P<0.001$ for rats 4 and 5 , respectively). These results are in agreement with previous findings that rats do not transfer to stimuli with inverted contrast polarity (Fields 1932; Lashley 1938). One possible explanation is that introducing a large bright region with the white background might be aversive for rats. A similar explanation might also account for failure to transfer to the Kanizsa shapes. However, the other possibility, explored later, is that the rats had been using a 
Group Learning Curve
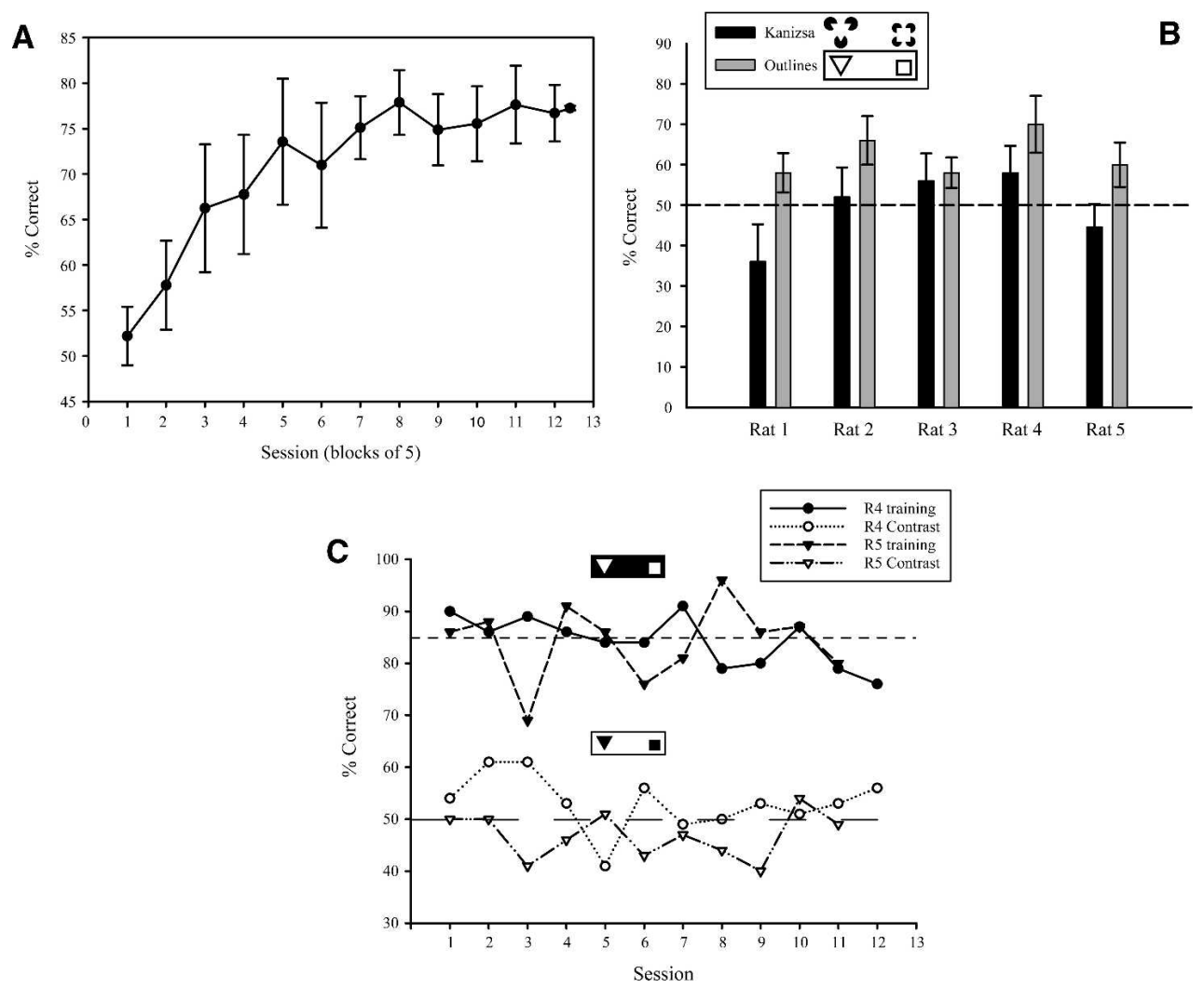

Figure 2. (A) The group learning curve (means \pm SEM) for training on the square-triangle discrimination (Experiment 1 ). (B) Individual transfer test performance (means \pm SEM) on the Kanizsa and Outline stimuli. For illustrative purposes the Outlines' stimuli in the graph are shown with reversed contrast polarity. The animals successfully transferred performance to the Outline stimuli but fell to chance or below for the Kanizsa stimuli. That this decrement may have been due to the reversal in contrast polarity is illustrated by the two rats shown in panel $C$, in which performance also fell to chance when the solid shapes were reversed.

luminance-based strategy that was disrupted by the reversal of luminance relationships in the Contrast stimuli.

Setting aside the question of whether or not the rats could perceive the illusory contours of the Kanizsa figures, we became curious as to how the rats were solving the basic discrimination task (the square-triangle discrimination they had been trained on). If the rats were using shape as the discriminative stimulus, they should perhaps show transfer to the same shapes at altered orientations. Correct discrimination performance for rotated stimuli might provide some (though not definitive) evidence that shape, rather than local low-level cues dependent on a specific retinal luminance distribution, was being used to solve the discrimination. The next probe tested this possibility.

\section{Probe 3: Tests for transfer to rotated stimuli}

Previous studies that directly examined generalization to rotated stimuli are not conclusive in this regard. Fields (1932) found successful transfer only to small rotations of $10^{\circ}$ to the left, but not to rotations of $20^{\circ}$ or more. Even after prolonged training with a larger stimulus set, transfer occurred only to rotations of $55^{\circ}$ to the left and up to $30^{\circ}$ to the right. Similarly, Lashley (1938) found that transfer failed when a triangle and cross were rotated by $90^{\circ}$ and $45^{\circ}$, respectively, although it succeeded when " $\mathrm{H}$ " and " $\mathrm{X}$ " were rotated by the same amount. Taken together, these results suggest that rats can transfer to rotated stimuli, but that this ability is largely modulated by the magnitude and side of the rotation and by the type of stimuli used. We explored this by presenting rats with the shapes at increasingly rotated orientations, to determine if there would be a point at which generalization failed (suggesting use of low-level cues) or if they were proficient at all rotations (suggesting use of shape).

In this set of probes we adopted a modified staircase procedure (Wetherill and Levitt 1965; see Materials and Methods), a standard method used to determine psychophysical thresholds in humans, to measure orientation invariance in rats. With the exception of rat 4, high rotation thresholds were obtained for all subjects, clearly indicating that these rats were able to solve the discrimination when even quite substantial changes to the orientation of the stimuli were introduced. Rats 1, 2, and 3 were able to solve the discrimination with stimuli rotated by $60^{\circ}$ (which maps the triangle onto itself). The threshold was similarly high for rat 5 , who passed the $56.25^{\circ}$ rotation level. Rat 4 was much lower at $26.25^{\circ}$. It should be noted that the $60^{\circ}$ rotation was the upper boundary of the rotation range used in this study, and that rats 1,2 , and 3 might therefore have reached a higher rotation threshold if further tested.

Taken together, these results at first glance seem to suggest that rats have some degree of orientation invariance and that their ability to discriminate between the two stimuli was largely independent of the specific orientation of the training set. It should also be noted that rotating the stimuli around their center also changed, to some extent, their spatial location. Thus, correct discrimination with these stimuli also suggests that rats have some degree of translational invariance; that is, that they can recognize objects irrespective of location. Evidence of orientation 
and translational invariance could have been interpreted as evidence of shape discrimination in this species. However, as explored in the next probe, a lowlevel explanation in fact more fully accounted for these results.

\section{Probe 4: Tests for use of lower hemifield features}

In the stepwise Rotation trials of Probe 3 , we were surprised by the observation that one rat suddenly reversed its shape preference between one step and the next. Because a trivial increment in rotation (illustrated in Fig. 1, Probe 3) produced such a striking reversal of shape preference, we looked carefully at what happened to the shapes at this transition point and noted that the critical rotation had the effect of rotating the target shape out of the lower hemifield. We thus speculated that this rat, and by implication perhaps the others too, might be using just the lower hemifield of the screen to make a judgement. This would be in accord with Lashley's (1938) finding, substantiated several times since (Sutherland 1961a; Simpson and Gaffan 1999), that rats prefer to use the lower parts of the shapes when making discriminations.

If the animals were in fact using the lower hemifield, in which the triangle had a vertex down and the square a base down, then reversing this relationship so that the triangle became base-down and the square vertex-down should cause a reversal in choice preference. Conversely, presenting both shapes with vertex-down or both with basedown should cause performance to fall to chance. Subjects were thus presented with Reflected-diamond stimuli (RD), in which the triangle was reflected on the $x$-axis to become base-down, and the square was presented in a diamond configuration, and with the Triangle-diamond (TD) and Reflectedsquare (RS) stimuli, where the square and triangle, respectively, were reflected on the $x$-axis and presented in pairing with the corresponding unrotated training shape (Fig. 1, Probe 4).

As shown in Figure 3, A and B, in the Reflected-diamond trials all subjects showed a preference for the negative shape, which was confirmed to be significant by a two-tailed onesample $t$-test $\left(t_{(3)}=4.90, P<0.05\right)$. Thus, during these test trials, subjects that had been previously trained to choose the square now chose the base-down triangle, and subjects that had been previously trained to choose the triangle now chose the vertexdown square. Remarkably, this behavior was observed for all animals. It thus seems they were ignoring the shape of the stimuli and using a local feature in the lower hemifield to make the discrimination. These conclusions were further supported by the level of performance observed in the Triangle-diamond condition, which was not significantly different from chance level $\left(t_{(3)}=1.89\right.$, NS). This suggests that subjects were no longer able to solve the discrimination when they were presented with two ver-
A

\begin{tabular}{cccc}
\hline Test stimuli & \multicolumn{1}{c}{ RD } & \multicolumn{1}{c}{ TD } & $\boldsymbol{\Delta}^{\text {RS }}$ \\
\hline Rat 1 & 7 & 50 & 47 \\
Rat 2 & 30 & 70 & 43 \\
Rat 4 & 27 & 90 & 43 \\
Rat 5 & 3 & 57 & 43 \\
Mean & 16.67 & 66.67 & 44.17 \\
(s.e.) & $(6.80)$ & $(8.82)$ & $(0.83)$ \\
\hline
\end{tabular}

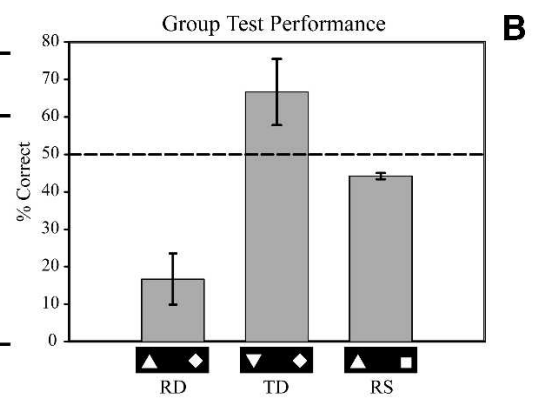

B

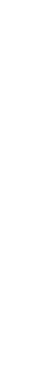

C
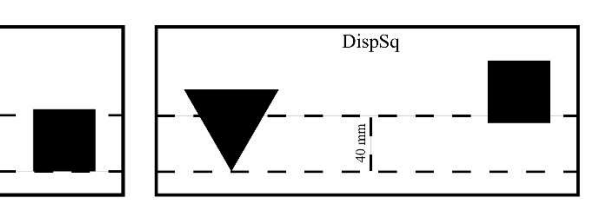

Group Test Performance

E

\begin{tabular}{|c|c|c|c|c|}
\hline & DispSq & DispBoth & Lum & DispTr \\
\hline Test stimuli & $\nabla$ & & $\nabla$ & - \\
\hline Rat 1 & 40 & 50 & 90 & 77 \\
\hline Rat 2 & 43 & 37 & 67 & 73 \\
\hline Rat 4 & 23 & 73 & 90 & 100 \\
\hline Rat 5 & 50 & 60 & - & _- \\
\hline $\begin{array}{l}\text { Mean } \\
\text { (s.e.) }\end{array}$ & $\begin{array}{l}39.17 \\
(5.67)\end{array}$ & $\begin{array}{l}55.00 \\
(7.76)\end{array}$ & $\begin{array}{l}82.22 \\
(7.78)\end{array}$ & $\begin{array}{l}83.33 \\
(8.39)\end{array}$ \\
\hline
\end{tabular}

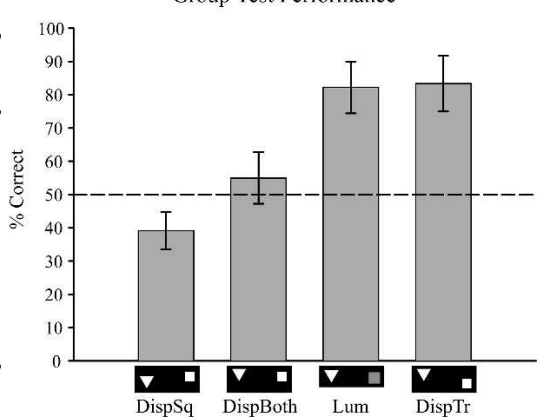

Figure 3. Individual $(A)$ and group $(B)$ performance with the stimuli testing the use of lower hemifield features. The preference for the negative shape can be clearly seen in the RD trials. (C) (For illustrative that the rats compared the shapes only below this $40-\mathrm{mm}$ level, which thus constitutes the uppe wn, the areas of the shapes that lay within the critical lower hemifield (between the dotted lines) reversal that their looking was guided by a touchscreen-based frame of reference rather than a shape-based one. Interestingly, when the luminance of the square in the critical region was reduced by dimming the shape rather than elevating it, performance remained high. Thus, although area is clearly a factor in the discrimination, this may be assessed on the basis of number of pixels rather than total brightness.

tices in the lower hemifield, even though the stimuli still consisted of a triangle and a square.

The ability to solve the discrimination was also disrupted in the Reflected-square trials, where two horizontal edges were presented in the lower hemifield. Interestingly, however, performance in this condition, instead of being at chance level was significantly below chance $\left(t_{(3)}=-7.00, P>0.01\right)$. After some consideration, it was realized that this behavior might be explained if subjects had used, as the discriminative cue, not the configuration of edges, but differences in the luminance of the two shapes in the lower region of the visual field. This possibility was further explored in the final probe set.

\section{Probe 5: Tests for use of lower hemifield luminance}

In order to test the luminance hypothesis, the vertical extent of the animals' processing-that is, the upper boundary of the "lower hemifield" - first had to be determined. The Reflectedsquare stimuli allowed us to estimate the location of this thresh- 
old to be at a $\sim 40$-mm vertical distance from the bottom edge of the two shapes. As shown in Figure 3C, at this level, the area of the triangle $\left(1812 \mathrm{~mm}^{2}\right)$ was greater than the area of the square $\left(1800 \mathrm{~mm}^{2}\right)$, but at a height of $41 \mathrm{~mm}$ this relationship reversed (area of triangle and square being 1833 and $1845 \mathrm{~mm}^{2}$, respectively). Given that subjects in the Reflected-square stimuli preferred the negative shape, the above calculations suggest that these rats may have computed the luminance of the two shapes below or at the 40-mm threshold.

This was tested by presenting the rats with Displaced-square (DispSq) stimuli, in which the square was elevated relative to the triangle so that it now possessed less area in the lower hemifield. Figure 3D shows that performance fell to chance $\left(t_{(3)}=-1.91\right.$, $P=0.07$ ), with a slight (not quite significant) preference for the negative shape. These results thus support the possibility that subjects used luminance differences in the lower hemifield to solve the discrimination task.

These conclusions are further supported by the results from the DispTr condition, where it was found that when the displacement of the triangle alone did not change the luminance differences in the lower hemifield, performance was maintained above chance level $\left(t_{(2)}=3.97, P>0.05\right.$, one-tailed; Fig. 3D). Thus, it can be seen that in this condition, unlike in the DispSq condition, there was a preference for the positive shape. These results rule out the possibility that the pattern of performance observed in the DispSq condition was the result of displacement alone.

To further test the lower-hemifield-luminance hypothesis, we presented rats with the Lum condition, in which the local luminance of the square was reduced to make the two shapes equiluminant below the $45-\mathrm{mm}$ threshold (corresponding to the height of the square). However, in this case performance was found, contrary to predictions, to be maintained at good levels $\left(t_{(2)}=4.14, P<0.05\right.$; Fig. 3D). This argues against our straightforward luminance hypothesis and raises the possibility that what the rats computed was not absolute luminance per se so much as the area of bright pixels, irrespective of the actual level of brightness.

Finally, the DispBoth condition tested whether the stimuli were processed relative to their position on the screen (allocentric coding) or to their position in the visual field (egocentric coding). As shown in Figure 3D, performance in this condition was at chance level $\left(t_{(3)}=0.65\right.$, NS, two-tailed) suggesting that a vertical displacement of both stimuli substantially disrupted performance. These results suggest that the two stimuli were encoded allocentrically, taking into consideration their position within the screen.

The results from Experiment $1 \mathrm{col}-$ lectively suggest that not only did rats not discriminate illusory shapes of the Kanizsa type, they did not use shape at all to solve the basic square-triangle discrimination, even though this cue was a readily available predictor of reward. Intrigued by this finding, in the next set of experiments we recruited a new group of rats to address the question of whether rats can be forced to process both dimensions simultaneously, in order to solve a shape-discrimination, if no other cues are available.

\section{Experiment 2}

In Experiment 2, changes to the training parameters prevented subjects from using the luminance-based response strategy they had used in Experiment 1. Four rats, assigned to the Bidimensional Discrimination (BD) group, were trained to discriminate between squares and rectangles selected from a very large stimulus set in which the two shapes varied in location and size. Relative height, relative width, and relative area were fully controlled so that aspect ratio was-we thought-the only constant discriminative cue. A control group of four rats, the Unidimensional Discrimination (UD) group, was trained to solve simpler squarerectangle discriminations that required relative size judgments only in the horizontal $(n=2)$ or vertical $(n=2)$ dimensions. These rats were used to confirm that rats can make each of the relevant unidimensional judgments, and to ascertain the learning curve thereof. For all animals, the size and location of the stimuli was continually changed during training. Thus, correct discrimination performance in any of these groups would also provide evidence of size and translational invariance in this species. The phases of training, together with illustrative stimuli from each set, are shown in Figure 4.

\section{Training}

Within-group analysis of performance for $\mathrm{BD}$ rats trained to choose squares versus rectangles revealed no difference dependent on which was the positive shape, so these data were combined. Similarly, in the UD group there was no difference dependent on whether the relevant dimension was horizontal or vertical, so these data were also combined. Figure $5 \mathrm{~A}$ shows the grouped learning curves for the BD and UD rats. Performance for the UD group increased at a faster rate than performance for the $\mathrm{BD}$ group $\left(t_{(34)}=-2.37, P<0.05\right.$, two-tailed $)$. However, some-

\begin{tabular}{|c|c|c|c|c|}
\hline \multicolumn{5}{|c|}{ Experiment 2} \\
\hline Phase & Purpose & Stimuli & \multicolumn{2}{|l|}{ Group } \\
\hline \multirow[t]{2}{*}{ Training } & $\begin{array}{l}\text { To establish shape discrimination } \\
\text { based on aspect ratio }\end{array}$ & $\begin{array}{l}\text { Square } v s \text { rectangle } \\
\text { (aspect ratio only } \\
\text { discriminand) }\end{array}$ & $\mathrm{BD}$ & \\
\hline & $\begin{array}{l}\text { To establish shape discrimination } \\
\text { based on the horizonal (UDH) } \\
\text { or vertical (UDV) dimension }\end{array}$ & $\begin{array}{l}\text { Square } v s \text { rectangle } \\
\text { (relative height, luminance } \\
\text { and position controlled) }\end{array}$ & $\begin{array}{l}\text { UDH } \\
\text { UDV }\end{array}$ & \\
\hline \multirow[t]{2}{*}{ Probe 1} & $\begin{array}{l}\text { To test transfer of responding } \\
\text { to stimuli with novel dimensions }\end{array}$ & $\begin{array}{l}\text { Generalization stimuli } \\
\text { (with novel dimensions) }\end{array}$ & $\mathrm{UDH}$ & E \\
\hline & & & UDV & \\
\hline \multirow[t]{2}{*}{ Probe 2} & $\begin{array}{l}\text { To test the possibility that rats } \\
\text { used aspect ratio }\end{array}$ & $\begin{array}{l}\text { No-discrepancy stimuli } \\
\text { (with no discrepancy in the } \\
\text { relevant dimension) }\end{array}$ & & \\
\hline & & & UDV & 口 \\
\hline \multirow[t]{2}{*}{ Probe 3} & $\begin{array}{l}\text { To test the possibility that rats } \\
\text { used area }\end{array}$ & $\begin{array}{l}\text { Area stimuli } \\
\text { (with equal area) }\end{array}$ & UDH & \\
\hline & & & UDV & \\
\hline
\end{tabular}

Figure 4. Summary of the training phases and illustrative examples of the stimuli used in Experiment 2. The Unidimensional Discrimination (UD) animals were divided into UDV, in which the stimuli differed only in the vertical dimension, and UDH in which they only differed in the horizontal dimension. The UD animals alone received probe tests to find out how they were making the discrimination. 
A Group Discrimination Performance

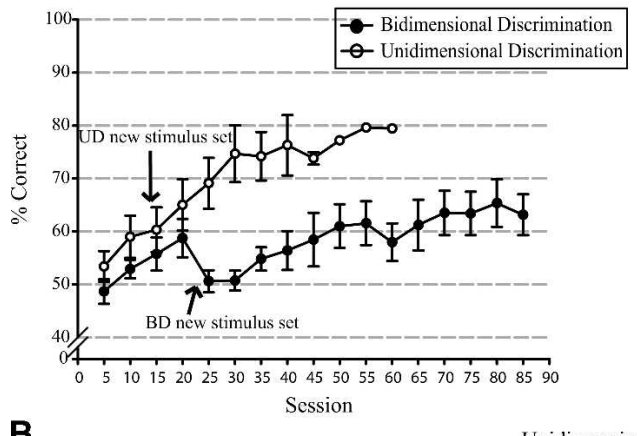

B Unidimensional Discrimination Test Performance C

\begin{tabular}{|c|c|c|c|}
\hline Test stimuli & & 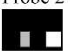 & \\
\hline Rat 5 & 61 & 33 & 69 \\
\hline Rat 6 & 75 & 47 & 50 \\
\hline Rat 7 & 67 & 31 & 67 \\
\hline Rat 8 & 61 & 50 & 56 \\
\hline $\begin{array}{l}\text { Mean } \\
\text { (s.e.) }\end{array}$ & $\begin{array}{l}66.00 \\
(3.32)\end{array}$ & $\begin{array}{l}40.25 \\
(4.82)\end{array}$ & $\begin{array}{r}60.50 \\
(4.52)\end{array}$ \\
\hline
\end{tabular}

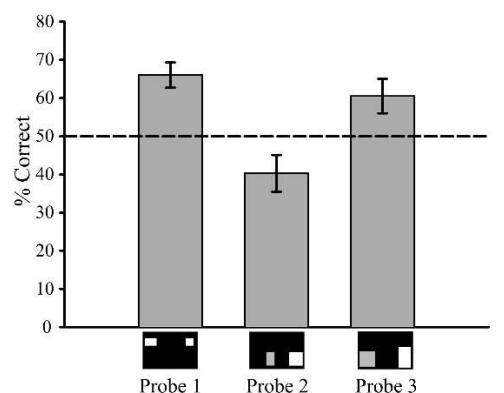

Probe 1 Probe 20 Probe 3

Figure 5. (A) Learning curves for the Bidimensional (BD) and Unidimensional (UD) groups. The graph illustrates performance up to criterion for the UD group and just before the testing phase for the BD group. Error bars indicate the standard errors. Individual $(B)$ and group $(C)$ performance in the three probes. (Arrows) Introduction of additional stimuli into the training sets. For the UD rats, stimuli of altered luminance were added after session 13 to discourage use of simple brightness as a discriminand. For the BD rats, intermediate-sized rectangles were added after session 18 to foil the use of an "avoid/choose extreme shapes" rule. Note that the rats fell back to chance at this point, suggesting they were indeed using a strategy other than shape to solve the task.

what surprisingly in view of our hypothesis, performance for the BD group in the first 18 sessions steadily increased to significantly above chance $\left(t_{(17)}=3.39, P<0.01\right.$, two-tailed). At this point we reviewed the stimulus set and realized that the discrimination could be made using the more complex "avoid/choose very large and very small shapes" rule discussed in the Introduction, and might thus require only a luminance or a unidimensional size judgment. We thus added new stimuli (medium-sized rectangles) to foil this strategy, at which point the BD rats, but not the control UD rats, fell to chance $\left(t_{(9)}=0.49\right.$, NS, two-tailed t-test for sessions 19-29; Fig. 5A). Thus, a clear difference in strategy use between the two groups had already developed by this stage.

Training continued until criterion performance for the control rats (on average for 26 sessions) and for a further 67 sessions for the BD rats. Given that each session comprised 128 trials, this resulted in $\sim 3330$ and 8600 trials for the UD and BD rats, respectively. To allow for the false start experienced by the BD rats, we compared the training curves (for 40 sessions) starting from session 1 for the UD rats and from session 25 for the BD rats. Even with this allowance, it can be clearly seen that there is a marked difference in the learning rate of the BD and UD groups, and statistical analysis confirmed that the control rats learned their discrimination significantly faster $\left(t_{(78)}=-5.99, P<0.001\right.$, twotailed). A $t$-test comparing asymptotic performance (last 15 sessions for the controls and 70-85 for the BD rats) confirmed that maximal performance was significantly worse for the rats in which aspect ratio was the only predictor of reward $\left(t_{(28)}=-9.25, P<0.001\right.$, two-tailed), being only $64 \%$ at asymptote. Thus, rats seemed to be quite poor at using the rule "choose the rectangle" or "choose the square," whereas they were quite good at using "choose the wider figure" or "choose the taller figure."

Despite the above, by the end of very prolonged training, performance for the $\mathrm{BD}$ rats was significantly above chance level, indicating that subjects were able to solve the discrimination to some extent. This was confirmed with a two-tailed one-sample $t$-test carried out on the last 10 training sessions $\left(t_{(9)}=20.53 ; P<0.001\right)$. These results suggest that rats might be somewhat capable of using aspect ratio, and by implication shape, to solve visual discriminations. However, the large number of trials needed to achieve levels of performance well below criterion suggests that this two-dimensional processing is not naturally used by rats to solve this type of task. These findings, together with the decrement in performance of $\mathrm{BD}$ rats when the new squares and rectangles were added to the stimulus set, further corroborate the conclusions from Experiment 1 that rats will try to solve shape discriminations using low-level cues, such as luminance differences, whenever possible.

The UD rats were able to use, in addition to aspect ratio, other discriminating features: particularly horizontal or vertical extent, and area. We were curious to know whether these animals had incidentally learned anything about the shapes of the stimuli or whether they were solving the discrimination task using the unidimensional size comparison alone. The animals were thus tested in three additional sets of probe trials.

\section{Probe 1: A test for generalization by the UD rats}

The first series of probes for these animals (Generalization stimuli; Fig. 4, Probe 1) tested their general ability to transfer to novel stimuli. Correct discrimination in this condition would have ruled out the possibility that these animals learned to respond to the specific dimensions of the training set (i.e., by using a "retinal snapshot"). A one-sample $t$-test revealed that correct responses with these stimuli were significantly above chance level $\left(t_{(3)}=4.82, P \leq 0.05\right.$; two-tailed), ruling out a snapshot strategy.

\section{Probe 2: A test for the hypothesis that UD rats might have used aspect ratio}

The use of aspect ratio as a discriminative cue for the UD rats was tested with the No-discrepancy stimuli (Fig. 4, Probe 2), which consisted of squares and rectangles that no longer differed in the training dimension, but that nevertheless maintained the same aspect ratio as in training. Good discrimination performance should have been observed if aspect ratio was used as a discriminative cue. In fact, these subjects performed at or below chance $\left(t_{(3)}=-2.02\right.$, NS; two-tailed), indicating that they had not spontaneously learned that aspect ratio (squareness or rectangleness) predicted reward. Interestingly, that their performance was actually slightly below chance suggests that they perhaps tried to transfer the unidimensional comparison that they had learned to the other dimension.

\section{Learning \& Memory \\ www.learnmem.org}




\section{Probe 3: A test for the hypothesis that UD rats might have used area} Because stimuli were always longer in one dimension and equal in the other, the rectangles for the UD group always had greater area than the squares, and so area may have formed, or helped form, the basis of successful discrimination for these animals. This was explored in the third series of tests (Areas stimuli; Fig. 4, Probe 3), in which subjects were presented with permutations containing squares and rectangles of equal areas. Performance did indeed fall to chance $\left(t_{(3)}=2.32\right.$, NS; two-tailed), suggesting the animals had been using area at least in part, and indicating, again, that aspect ratio was not spontaneously used in this task.

\section{Discussion}

The original aim of this study was to investigate whether rats perceive illusory contours, by measuring transfer of performance to Kanizsa illusory figures after training with solid shapes (a white square vs. a white triangle on a black background). In Experiment 1 , we found that not only could subjects not solve the square-triangle discrimination with the illusory figures, they had not been using shape at all to solve the discrimination. Instead, they relied on a more low-level cue: namely, luminance differences in the lower hemifield. When these differences were reversed, as in the Reflected-diamond and Displaced-square stimuli, a significant preference for the negative shape was observed. These results suggest that subjects did not use "squareness" or "triangleness" to solve the task but instead responded to the shape with the smallest/largest luminance in the lower portion of the visual field. The preference for processing visual stimuli in the lower hemifield observed in this study has also been reported by other authors (Lashley 1938; Sutherland 1961b; Simpson and Gaffan 1999).

It has been brought to our attention that the task in Experiment 1 could have measured detection rather than discrimination abilities. Specifically, that if the shape with the lowest luminance in the lower hemifield fell below the rat luminance detection threshold, then subjects could have learned to respond to the presence/absence of the stimuli at the target locations rather than to discriminate between them. It is important to note that a detection account would be plausible only if the luminance of the stimuli was computed in the lower region of the visual field and not in the whole visual field, as equiluminant stimuli were used in the study. Thus, the first main finding that luminance is processed only for a portion rather than the whole of the visual field would be supported by this new account. Moreover, the other main finding that luminance differences rather than shape information were used to solve the task would also be true for this second account. The only difference would be in the subject of the comparison; whereas the discrimination account suggests that luminance differences are compared across the two stimuli, the detection account claims that they are compared across the two target locations. Although it seems unlikely that in the training set the luminance of the triangle in the lower visual field fell below the rat luminance detection threshold, the present results cannot rule out this account. Future studies should directly address this question. In any case, either explanation would support the conclusion that subjects used luminance differences in the lower portion of the visual field to solve the task, even when shape information was readily available.

These results led us to explore whether rats could nevertheless be forced to process shape holistically if this were the only way to make the discrimination. Experiment 2 used a procedure that controlled for the use of several low-level featural response strategies, including the use of lower hemifield luminance. We thus tested the rats' ability to discriminate squares from rectangles, shapes that differ only in aspect ratio, which is the sim- plest "global" descriptor of shape, and one that is not confounded by featural differences such as angle acuity. For the Bidimensional Discrimination (BD) group, aspect ratio was the only relevant discriminand, and rats learned extremely slowly and reached asymptote at levels not much above chance. By contrast, rats in the control, Unidimensional Discrimination (UD) group, in which the squares and rectangles differed in one (consistent) dimension only, had no difficulty learning the discrimination, illustrating that the similarity of the shapes was not, in itself, a problem. These results suggest that aspect ratio, and by implication shape, is not naturally processed by rats in the solving of visual shape discriminations. This conclusion is further supported by the observation that the UD group did not learn to use aspect ratio even though this was readily available as an additional cue (revealed by the No-discrepancy and Area probes), and that the BD group spontaneously adopted a luminancebased response strategy when this was inadvertently allowed in the first 18 sessions of training; that is, they preferred the more complex unidimensional size-based rule "avoid/choose shapes that are very large or very small" than the simpler, shape-based rule "avoid/choose the rectangles." Taken together, these results suggest that rats that are successfully discriminating shapes may not in fact be processing these holistically, but using unidimensional features such as length or local brightness instead. This finding has important implications for researchers planning to use a visual discrimination task to explore perceptual processes.

\section{Prior shape discrimination findings}

Our findings surprised us somewhat, because we understood at the beginning of this study that shape-based shape discrimination was well established in rodents. Shape discrimination was explored extensively in the first half of the last century, and it was concluded from this body of work that a variety of species, including rats and mice, fish, octopi, and birds are capable of discriminating shapes with relative ease. The field was summarized by Sutherland's renowned monograph "Shape Discrimination by Animals" (Sutherland 1961a), which established that animals cannot only discriminate shapes, but can also transfer this discrimination to shapes that are transformed in a variety of ways (including rotation, translation, and conversion to outline forms). Some puzzling exceptions were noted, however. Lashley (1938), for example, noted that rats had great difficulty discriminating squares from circles, which seems surprising, but becomes less so if one considers the low visual acuity of rats (see below).

Notwithstanding these mostly clear findings, in few of these studies was the actual mechanism of shape discrimination explored in detail, except for Lashley's (1938) observation of lower hemifield selectivity, which we also found, and which has been replicated a number of times. In a number of cases, generalization on transfer tests was taken as evidence of holistic shape processing. However, as we found in the present study, generalization does not prove perceptual shape constancy. In our study, although we too found successful transfer to rotated shapes, a chance observation (of sudden preference reversal following an apparently trivial rotational increment) led us to determine that the animals had been using lower hemifield luminance rather than shape to make their discrimination.

Our finding that rats have a strong preference for using luminance differences rather than shape to solve this type of visual discrimination is in agreement with a recent study by Young and colleagues. Young et al. (2001) trained pigeons to discriminate between two-dimensional renderings of three-dimensional objects that included realistic illumination cues. The authors found that subjects responded above chance level with silhouettes or when the illumination cues were changed; however, perfor- 
mance significantly decreased in both conditions relative to training. The ability to solve the task above chance level with silhouettes suggests that to some degree shape information was used. However, the significant decrement in performance observed with illumination changes also indicates that luminance cues played a major role. Chance level performance recorded with line drawings confirmed that shape information alone was not sufficient for correct discrimination. Further exploration of which luminance cues were used by the animals revealed that changes to the relative luminance of the object's surfaces, but not changes to the object's overall luminance, impaired performance. Young et al.'s (2001) findings suggest that, as we found for rats, pigeons are sensitive to and use relative luminance differences to solve visual discriminations, even when shape information would be a better predictor of reward. These results are of interest as, unlike rats, pigeons are highly visual animals with good vision. Thus, the preference for luminance cues observed in this study does not seem to be a strategy exclusively used by animals for whom vision does not provide a primary source of sensory information.

\section{Can rats perceive shapes holistically?}

Since our rats apparently did not use global shape to make their discriminations, the question arises as to whether they can actually holistically perceive shapes at all. Some sparse but converging evidence supports the possibility that perhaps they cannot. First, it is worth recalling that rats have quite poor visual acuity compared with humans, and see as well at $1 \mathrm{~m}$ as we see at $10 \mathrm{~m}$. Thus, shapes that appear quite clear to us would seem blurred to a rat (see http://www.ratbehavior.org/RatVision.htm for an excellent illustration of this). Second, rats have been found to have a diminished capacity, relative to humans, to use proximity and alignment cues for perceptual grouping (Kurylo et al. 1997). Kurylo et al. (1997) found that rats trained to discriminate horizontal from vertical solid luminance-defined lines did not readily transfer to arrays of similar orientations that consisted of disjoint elements (dots) varying in proximity and alignment. Specifically, Kurylo et al. (1997) found that proximity was used as a cue for perceptual grouping; however, its effectiveness was diminished relative to humans. Moreover, alignment was not used by rats as a grouping cue when tested in isolation. A diminished ability to use proximity and alignment cues for perceptual grouping would likely result in poorer object recognition in this species. In particular, it would diminish these animals' ability to use shape as a discriminative cue.

\section{Can rats discover "shape" as the relevant discriminand in a simultaneous discrimination task?}

Notwithstanding the above, it seems intuitively unlikely that rats, with a highly developed visual system not unlike our own, do not have form vision as such. This intuition is supported by recent findings that the rat visual system is more developed and differentiated than previously proposed (Montero 1993; Girman et al. 1999).

If it turns out that rats can indeed perceive shapes holistically, why then did they not seem to do so in our tasks? The most likely possibility is that rats do not spontaneously discover shape to be the relevant discriminand in a simultaneous discrimination task. Relatively little is known about the detailed mechanisms by which animals make discriminations, partly because of the difficulty of conducting the elaborate set of probe tests necessary to determine the mechanisms behind their performance. In particular, the way in which animals use combined sets of features remains relatively unexplored (George and Pearce 2003). The recent development of touchscreen technology offers the ability to conduct large numbers of trials with relative ease. Using this technology we were able to give our animals extensive probe testing, and to show that irrespective of whether they have the capacity for form vision, they did not seem to exercise it in our task. We believe this may reflect the constraints of discrimination tasks rather than a limitation in rat vision. When a rat is faced with a simultaneous discrimination, it needs to discover, by experimentation, which is the relevant stimulus dimension, and it may be that the two-dimensional parameter of global shape is less easily discovered than unidimensional parameters such as brightness and unidimensional size. Furthermore, the predilection, replicated in a number of studies, for rats to process only the lower visual hemifield suggests that they do not naturally tend to try and process the entirety of a visual object. Indeed, ecological considerations suggest that rats probably prefer to discriminate objects by olfaction rather than vision. Taken together, our results suggest that while simultaneous visual form discrimination tasks remain very useful in probing the mechanisms behind simultaneous discrimination, they are less likely to be useful for those interested in the neurobiology of higher-level visual perceptual processes.

\section{Materials and Methods}

\section{Subjects}

\section{Experiment 1}

Five experimentally naive male Hooded Lister rats, weighing between 510 and $540 \mathrm{~g}$ at the beginning of the experiment, were kept on a 12-h light/12-h dark cycle and between $85 \%$ and $90 \%$ of their free-feeding weight. All animals had free access to water in the home cages and were housed in groups of two and three.

\section{Experiment 2}

Dark Agouti rats were used in this experiment because of their superior performance, relative to Lister Hooded, in concurrent visual tasks (Gaffan and Eacott 1995; Gaffan and Woolmore 1996). Eight experimentally naive males, weighing between 215 and $255 \mathrm{~g}$ at the beginning of the experiment, were kept on a 12-h light/12-h dark cycle and at $85 \%$ of their free-feeding weight. All animals had free access to water in the home cages and were housed in groups of four. Four of the animals were in the experimental (Bidimensional Discrimination) group and four were in the control (Unidimensional Discrimination) group.

\section{Apparatus}

In all experiments, we used an automated touchscreen apparatus to present stimuli at one end of a rectangular box and rewarded the animals for correct choices with a pellet dispenser at the other end. The aluminium box was the size of a standard manual dual-discrimination box (height $=30.5 \mathrm{~cm}$; length $=89 \mathrm{~cm}$; width $=45 \mathrm{~cm}$; all internal dimensions). It had a lid in transparent Perspex and a removable floor. The internal aluminum parts were painted matte black. One end of the box consisted of a 17" CRT flat screen VGA monitor (Mitsubishi, Diamond Pro 710; resolution $800 \times 600$ ) covered with a pressure-sensitive glass panel (17" IntelliTouch, Elo TouchSystems) positioned so that the screen started $1 \mathrm{~cm}$ above the floor. In the end opposite the touchscreen, a standard magazine tray with a hinged Perspex door was positioned $1 \mathrm{~cm}$ above the floor, attached to a standard pellet dispenser (Campden Instruments Ltd.). A $24 \mathrm{~V} 2.8 \mathrm{~W}$ M.E.S. single filament lamp was placed approximately at the center on the lid (house light), and a speaker was positioned adjacent to it. Another similar lamp was positioned inside the magazine tray, and a third $10 \mathrm{~cm}$ above the magazine tray. This latter was not used in any of the procedures reported here. The touchscreen, lights, speaker, and pellet dispenser were connected to a standard PC via custom-made hardware and were operated with custom-made software.

In Experiment 2, a partition $(30 \times 15 \mathrm{~cm})$ divided the 
screen in two halves. This was introduced to force animals to make a choice before reaching the touchscreen, and therefore prevented side-switching at the response location.

\section{Stimuli for Experiment 1}

See Figure 1 for illustrations of these, which had characteristics as follows:

\section{Training stimuli}

These consisted of a white $\left(83 \mathrm{~cd} / \mathrm{m}^{2}\right)$ square and equilateral triangle with the vertex down. The two shapes had equal area and equal local luminance and were presented on a black $(0.03 \mathrm{~cd} /$ $\mathrm{m}^{2}$ ) background. During the automated pre-training procedure, the size of the square and triangle systematically decreased from, respectively, $85 \times 85$ and $119 \times 129 \mathrm{~mm}, 65 \times 65$ and $86 \times 99$ $\mathrm{mm}, 45 \times 45$ and $59 \times 68 \mathrm{~mm}$.

\section{Probe 1: A test for the perception of illusory figures}

Test stimuli consisted of a Kanizsa triangle and a Kanizsa square of the same dimensions as the training stimuli. Because the illusory shapes were intended to seem white, the presence of the black inducers (the notched circles that define the "shape") necessarily required a white background. The inducers had a diameter of 23 and $34 \mathrm{~mm}$ for the square and triangle, respectively.

\section{Probe 2: Tests for transfer to altered stimuli}

The Contrast stimuli were obtained by inverting the contrast polarity of the training stimuli so that black shapes were now presented on a white background. The Outline stimuli were obtained by superimposing (with centers aligned) a black $31 \times 31$ $\mathrm{mm}$ square and a $41 \times 47-\mathrm{mm}$ equilateral triangle on the training square and triangle. These dimensions ensured that the area of the white border region was the same for the square and triangle.

\section{Probes 3: Tests for transfer to rotated stimuli}

The Rotated stimuli were obtained by rotating, clockwise and around their center, the training stimuli by incremental steps of $3.75^{\circ}$. Rotations ranged from $0^{\circ}$ to $60^{\circ}$.

\section{Probe 4: Tests for use of lower hemifield features}

The Reflected-diamond stimuli were obtained by rotating the training triangle and square by $60^{\circ}$ and $45^{\circ}$, respectively. The Triangle-diamond and Reflected-square stimuli were identical to the Reflected-diamond stimuli except that the square and triangle, respectively, were left as in training. In these three conditions, the stimuli were rotated around their center, but their position along the vertical axis was subsequently adjusted to maintain a $20-\mathrm{mm}$ distance from the bottom edge of the screen as during training.

\section{Probe 5: Tests for use of lower hemifield luminance}

In the first set, the Displaced-square stimuli (DispSq), the luminance relationship of the two shapes below the critical $40-\mathrm{mm}$ threshold was reversed by vertically displacing the square by 35 $\mathrm{mm}$, so that the area of this shape below this threshold was, unlike training, smaller than the area of the triangle.

The Lum stimuli were obtained by reducing the local luminance of the square to $47.25 \mathrm{~cd} / \mathrm{m}^{2}$ so that its global luminance matched the global luminance of the triangle (local luminance $81.84 \mathrm{~cd} / \mathrm{m}^{2}$ ) below the $45-\mathrm{mm}$ threshold. In a CRT monitor, luminance levels can vary according to several factors (Harris et al. 1987); therefore, luminance values were calculated as the average of three measurements.

The Displaced-triangle (DispTr) stimuli controlled for the possibility that displacement alone could have been sufficient to disrupt performance in the DispSq condition. In the DispTr stimuli, the triangle was vertically displaced by $35 \mathrm{~mm}$; however, because the luminance relationship between the two shapes below the 40-mm threshold was not changed relative to training, no disruption in performance should have been observed in these trials. In contrast, if the effect observed in the DispSq con- dition was the result of displacement alone, a similar effect should have been observed in the DispTr condition.

The Displaced-both (DispBoth) stimuli examined whether the two shapes were processed relative to their position on the screen (allocentric coding) or relative to their position in the visual field (egocentric coding). Both shapes were thus vertically displaced by $35 \mathrm{~mm}$.

\section{Stimuli for Experiment 2}

\section{Training stimuli}

\section{Bidimensional Discrimination (BD) group}

For this group, every display (or permutation) consisted of a white square and a white $\left(83 \mathrm{~cd} / \mathrm{m}^{2}\right)$ vertically oriented rectangle presented on a black $\left(0.03 \mathrm{~cd} / \mathrm{m}^{2}\right)$ background. Two sets of stimuli were used; one was used from the start of the study and the other was introduced after 18 sessions. Briefly, the stimuli varied in size from $15 \times 15-90 \times 90 \mathrm{~mm}$ (squares) and $50 \times 25$ $80 \times 40 \mathrm{~mm}$ (rectangles). The screen was subdivided into two halves containing four identical quadrants, and, within the set, each of the eight shapes was presented once in every quadrant. Stimuli were positioned at the center of the quadrants, with adjustments where necessary for the larger figures.

The dimensions of the squares and rectangles ensured that the relative height, width, and area of the two shapes were fully controlled within the stimulus set. The square was higher, wider, and of greater area than the rectangle in half of the permutations, and it was shorter, narrower, and smaller in the other half. This ensured that these low-level properties could not be used to solve the discrimination. Each of the 32 permutations was presented four times in a training sequence of 128 trials (one session). The position of the permutations in the training sequence was determined by a random function.

As noted in the introduction to this experiment, we discovered after 18 sessions that to maintain the relative size constraints outlined above, permutations always contained averagesized rectangles paired with large or small squares, and that rats might be using this unanticipated regularity to solve the discrimination on the basis of the rule "avoid/choose very large and very small shapes." To control for this possibility, a new set of average-sized squares and very large and very small rectangles was introduced. These stimuli were constructed as described above and yielded 32 new permutations that, together with the initial set, were now presented twice in each training sequence.

\section{Unidimensional Discrimination (UD) control groups}

The Unidimensional Discrimination control groups were subdivided into a Unidimensional Discrimination Horizontal (UDH) group $(n=2)$ and a Unidimensional Discrimination Vertical (UDV) group $(n=2)$. For the UDH group, a square with a side of $30,40,50$, or $60 \mathrm{~mm}$ was always paired with a rectangle that had twice its width, whereas for the UDV group these squares were paired with rectangles that had twice their height. For both groups, the background was maintained black; however, in order to prevent the use of either local or global luminance as a discriminative cue, the luminance of the square and rectangle varied among three possible luminance levels. The first luminance set was presented from the start of training and contained white squares and rectangles $\left(83 \mathrm{~cd} / \mathrm{m}^{2}\right)$. For the two UD groups the rectangle had always twice the area of the square, and after 13 sessions it was realized that subjects could have used this systematic difference in global luminance to solve the discrimination. This possibility was controlled for by introducing two additional luminance levels.

Each luminance set resulted in 32 permutations, making 96 in total that were presented once in the 128-trial training sequence. For the remaining 32 trials, 11 permutations were randomly taken from the first luminance set, 11 from the second, and 10 from the third.

\section{Probe stimuli}

The Generalization stimuli, which tested for generalization, were obtained by pairing four squares with a side of $15,20,25$, and 70 
mm with matching rectangles of equal height and double width for the UDH group, and of equal width and double height for the UDV group. A total of 12 permutations, four for each of the three luminance levels used in training, was constructed for this set of probes. Permutations were formed with a procedure similar to that used for the training set.

For the UDH group, the No-discrepancy stimuli, which tested for the use of aspect ratio, were obtained by pairing four squares with a side of $50,60,70$, and $80 \mathrm{~mm}$ with rectangles of the same width and half the height. Thus, for these stimuli the square and rectangle did not differ in the previous relevant (horizontal dimension), but maintained the same aspect ratio as in training. For the UDV group, the same squares were paired with rectangles of the same height and half the width, resulting in pairs that did not differ in the vertical dimension. The rest of the procedure was as for the Generalization stimuli.

Finally, for the UDH group, the Area stimuli, which tested for the use of area, were obtained by pairing four squares with a side of $40,50,60$, and $70 \mathrm{~mm}$ with rectangles of the same area and aspect ratio as used in training. For the UDV group, the same permutations were used except that the rectangles were rotated by $90^{\circ}$. The rest of the procedure was as for the Generalization stimuli.

\section{Procedure: Experiment 1}

Three rats were assigned the square as the positive shape and two rats the triangle.

\section{Pre-training}

Over an 8-d period, rats were gradually habituated to the apparatus and shaped to approach the touchscreen on presentation of the positive shape, and then return to the magazine tray, at the other end of the box, for food reward.

\section{Training}

The automated training consisted of five stages during which the sizes of both stimuli and responsive area on the touchscreen were systematically reduced. Progression from one stage to the next occurred when the animals reached asymptotic performance. Correct responses (nose pokes to the responsive area) were followed by stimulus offset, a $1-\mathrm{sec} 4-\mathrm{KHz}$ tone, the onset of the magazine light that remained on until the next stimulus presentation, and the delivery of one 45-mg food pellet in the magazine tray. Nose pokes outside the responsive area (incorrect responses) were followed by stimulus offset and a 10-sec blackout period. Stimulus offset was triggered by a response (either correct or incorrect), the ITI was 10-sec, and sessions lasted for $30 \mathrm{~min}$. Except during blackout periods, the house light remained switched on and provided the only source of light in the room. The fan of the PC provided background noise, and the floor of the apparatus and touchscreen were cleaned between animals.

\section{Probe testing}

\section{Kanizsa}

Testing began once performance reached asymptote. Performance with the Kanizsa stimuli was collected over five sessions each containing 10 test trials interleaved with 130 training trials. Test trials could not occur in the first five trials and were separated by between five and nine training trials.

\section{Contrast}

After testing with the Kanizsa condition, rats 4 and 5 were given additional training sessions until they reached asymptotic performance. After this, they were presented with sequences in which half of the training trials were interleaved with the Contrast stimuli. Stimuli were presented randomly with the only constraint that each of the four shape $\times$ contrast-polarity combination occurred at the left and right response locations with equal probability (25\%). This procedure was carried out for 12 sessions for rat 4 but had to be interrupted after 11 sessions for rat 5 , as at this point this subject developed an aversion to the door of the magazine tray.
Outline

Rats 1,2, and 3 were tested with the Outline stimuli directly after the Kanizsa trials. All five animals were presented with training sessions and brought to asymptotic performance before testing began. After a number of attempts to retrain rat 5, it was decided to try the procedure with the magazine door taped open. Performance increased immediately to asymptotic level, and this modification was therefore adopted for all the subsequent trials with this animal. All subjects were tested with the Outline stimuli for five consecutive sessions.

\section{Rotation}

After the Outlines trials, all rats were presented with training sessions until they reached asymptotic performance and then were tested with the Rotated stimuli. The two-up-two-down staircase procedure (Wetherill and Levitt 1965) was applied iteratively, starting at $30^{\circ}$, until a threshold level was confirmed over two phases, and it allowed a quick determination of rotation thresholds (within 3-6 phases) for all subjects except for rat 3, who was withdrawn from the study due to deteriorating health. For this subject, the threshold was based on the measurement obtained from a single phase. The staircase started with a $30^{\circ}$ rotation, and the angle of rotation was increased if $\geq 70 \%$ correct performance was achieved over two consecutive sessions for the same level. The angle of rotation was decreased if this criterion was not reached. Movements up and down the staircase were always of two steps unless this led to a previously visited level, in which case increments and decrements of one step were used. Phase 1 ended when movements of one step led to a previously visited level. An identical procedure was used for successive phases except that the start point was determined by performance in the previous phase. Specifically, the new phase would start one level up or at the same level if the last level of the previous phase was passed or failed, respectively. If the same level was failed twice over two consecutive phases, the new phase would start one level down. For all phases, increments and decrements from the start point $\left(30^{\circ}\right)$ were always of four steps. Individual phases were separated by three training sessions.

\section{Luminance}

After the staircase procedure, all rats were presented with training sessions until they reached asymptotic performance. After this, they were presented with three sessions for each of the RD (Reflected-diamond), TD (Triangle-diamond), and RS (Reflectedsquare) stimuli, respectively. All test sessions were alternated with training sessions. Given the large number of non-reinforced test trials that had been presented to the animals at this point of the study, the same contingencies used in training were adopted for these and all the subsequent test trials.

After the RD, TD, and RS test trials, subjects were presented with training sessions until they reached asymptotic performance. Subjects were then tested with the DispSq (Displacedsquare), DispBoth (Displaced-both), Lum, and DispTr (Displacedtriangle) stimuli, respectively. Each set of stimuli was presented for three sessions that were alternated with training sessions. For health reasons, rat 5 was withdrawn from the study after the DispBoth condition.

\section{Procedure: Experiment 2}

\section{Training}

Over three days, rats were first habituated to the apparatus and introduced to the magazine procedure. Training with the stimuli and touchscreen started on day 4 .

Unless otherwise stated, the same procedure was used for all groups. In the BD (Bidimensional discrimination) group, half of the subjects were randomly assigned the rectangle as the positive stimulus (rats 1 and 2) and the other half the square (rats 3 and 4). Similarly, for the UDH (Unidimensional discrimination horizontal) group, rat 5 was assigned the rectangle as the $\mathrm{S}+$ and rat 7 the square. For the UDV (Unidimensional discrimination vertical) group, rat 6 was assigned the rectangle as the $S+$ and rat 8 
the square. The stimuli remained on the screen until the animal nose poked the touchscreen. Nose pokes to the hemiscreen with the $\mathrm{S}+$ were coded as correct responses and were followed by stimulus offset, onset of the light inside the magazine tray, and delivery of one 45-mg food pellet. Stimulus offset resulted in a black screen. Nose pokes to the hemiscreen with the $S$ - were coded as incorrect responses and were followed by stimulus offset, a 2 -sec burst of white noise, and a 10-sec blackout period. For the initial 30 sessions, the ITI after correct responses was set at 3 $\mathrm{sec}$, but this was reduced to $1 \mathrm{sec}$ thereafter. A modified correction procedure was used whereby the same permutation was repeated up to two times after an incorrect response. Responses from correction trials were not included in the calculation of the percent correct performance. Training sessions lasted for 128 trials. Background noise was provided by the fan of the PC, and the floor of the apparatus and touchscreen were cleaned between animals.

\section{Testing}

Testing started immediately after the animals reached criterion performance ( $\geq 80 \%$ correct over three consecutive sessions). Unless otherwise stated, the same procedure was used for all test conditions. In test sessions, the 128-trial sequence contained 12 probe permutations pseudorandomly interleaved with 116 training trials. Test trials could not occur in the first five trials of the sequence and were separated by between five and nine training trials. For all tests (Generalization, No-discrepancy, and Area), each of the 12 probe permutations was presented once. The contingencies after correct and incorrect responses for test trials were maintained as in the training trials, with the exception that the modified correction procedure was not used for incorrect responses to test stimuli. Each set of probes was presented for three consecutive sessions and testing progressed from the Generalization to the No-discrepancy to the Area trials.

\section{Acknowledgments}

We thank John Wattam-Bell for many helpful discussions and assistance and for suggesting the Lum stimuli as a test condition, John Pearce and Mark Good for helpful comments on the manuscript, Roger Bunce and Trevor Brooks for the construction of the apparatus, and Preetha Shaji and Martin Donovan for assistance with the control software. This work was supported by a BBSRC grant to K.J. and a Departmental Demonstratorship to L.M.

\section{References}

Bussey, T., Muir, J.L., and Robbins, T.W. 1994. A novel automated touchscreen procedure for assessing learning in the rat using computer graphic stimuli. Neurosci. Res. Commun. 15: 103-110.

Bussey, T.J., Saksida, L.M., and Rothblat, L.A. 2001. Discrimination of computer-graphic stimuli by mice: A method for the behavioral characterization of transgenic and gene-knockout models. Behav. Neurosci. 115: 957-960.

Cook, R.G., Geller, A.I., Zhang, G.R., and Gowda, R. 2004. Touchscreen-enhanced visual learning in rats. Behav. Res. Methods Instrum. Comput. 36: 101-106.

Fields, P.E. 1932. Studies in concept formation. The development of triangularity by the white rat. Comparative Psychology Monographs 9: $1-70$.

Gaffan, E.A. and Eacott, M.J. 1995. A computer-controlled maze environment for testing visual memory in the rat. J. Neurosci. Methods 60: 23-37.

Gaffan, E.A. and Woolmore, A.L. 1996. Complex visual learning by rats. Learn. Motiv. 27: 375-399.

George, D.N. and Pearce, J.M. 2003. Discrimination of structure: II. Feature binding. J. Exp. Psychol. Anim. Behav. Process. 29: 107-117.

Girman, S.V., Sauvé, Y., and Lund, R.D. 1999. Receptive field properties of single neurons in the rat primary visual cortex. J. Neurophysiol. 82: 301-311.

Harris, J.P., Makepeace, A.P., and Troscianko, T.S. 1987. Cathode ray tube displays in psychophysiological research. J. Psychophysiol. 1: 413-429.

Kurylo, D.D., Van Nest, J., and Knepper, B. 1997. Characteristics of perceptual grouping in rats. J. Comp. Psychol. 111: 126-134.

Lashley, K.S. 1938. The mechanism of vision: XV. Preliminary studies of the rat's capacity for detailed vision. J. Gen. Psychol. 18: 123-193.

Montero, V.M. 1993. Retinotopy of cortical connections between the striate cortex and extrastriate visual areas in the rat. Exp. Brain Res. 94: $1-15$.

Peterhans, E. and von der Heydt, R. 1991. Subjective contours: Bridging the gap between psychophysics and physiology. Trends Neurosci. 14: $112-119$.

Simpson, E.L. and Gaffan, E.A. 1999. Scene and object vision in rats. Q. J. Exp. Psychol. 52B: 1-29.

Sutherland, N.S. 1961a. Shape discrimination by animals. Experimental Psychology Society Monographs, Heffer and Sons, Cambridge, UK.

. 1961b. Visual discrimination of horizontal and vertical rectangles by rats on a new discrimination training apparatus. Q. J. Exp. Psychol. 13: 117-121.

Wetherill, G.B. and Levitt, H. 1965. Sequential estimation of points on a psychometric function. Br. J. Math. Stat. Psychol. 18: 1-10.

Young, M.E., Peissig, J.J., Wasserman, E.A., and Biederman, I. 2001. Discrimination of geons by pigeons: The effects of variation in surface depiction. Anim. Learn. Behav. 29: 97-106.

Received September 12, 2005; accepted in revised form February 6, 2006. 


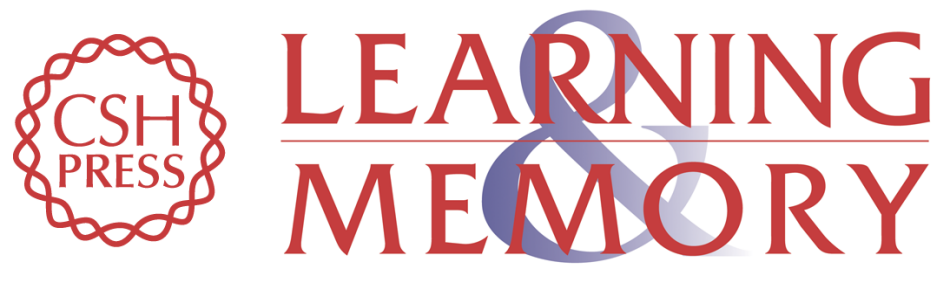

\section{Do rats use shape to solve "shape discriminations"?}

Loredana Minini and Kathryn J. Jeffery

Learn. Mem. 2006, 13:

Access the most recent version at doi:10.1101/lm.84406

\section{License}

Email Alerting Receive free email alerts when new articles cite this article - sign up in the box at the Service top right corner of the article or click here. 